\title{
Hardware Implementation of a Real Time Image Compression
}

\author{
Muzhir Shaban Al-Ani \\ University of Human Development, College of Science and Technology, Department of Computer Science, \\ Sulaimani, KRG, Iraq [E-mail: muzhir.al-ani@uhd.edu.iq]
}

\begin{abstract}
Real time processing of image deals with applying all required operations within a range of time not exceed the acceptable time of human eyes. Real time processing does not realized on standalone computer, so it need special hardware. The big challenge of image processing work is the processing time, in which there are big amount of data (pixels) that must be processed at a specific time. The most important objective aspect of this work and any image processing algorithm (software or hardware) is how to implement it in an efficient way via the effective management of the scheduling of the processing jobs and time allocated for each job. This work try to avoid this problem by inserting a Raspberry Pi device which is working as microcomputer and has the ability to work with image, video, audio and data. Compensating between hardware and software is very important to achieve an efficient implemented system. This work mixing between hardware (Raspberry Pi device) and the software compression algorithm to reach acceptable low cost and high speed operated system. The synchronization between hardware and software leads to an efficient architecture that can achieve the real time processing.
\end{abstract}

Keywords: Image Compression, 2D-DWT, Real Time Processing and Image Hardware Implementation.

\section{Introduction}

Discrete Wavelet Transform (DWT) becomes more popular in many fields and it have wide applications in digital image compression [1,2,3]. Many filters for compression were introduced many masks of DWT and one important application is JPEG2000 standard [4,5,6]. DWT is performed via the implementation of low pass filter (LPF) and high pass filter (HPF) on the original data $[7,8,9]$. DWT has proven useful in the area of image compression where it replaces the Discrete Cosine Transform (DCT) in new JPEG2000 and MPEG4 image and video compression standards $[10,11,12]$. DWT is an efficient tool for data compression and on of special hardware application is neural interface $[13,14,15]$. DWT cores using convolution require two passes per row/column of pixels for each 1-D DWT that is performed $[16,17]$. DWT cores using the lifting scheme require two or more passes $[18,19]$. This wavelet core implements the "line-based" DWT similar to that described in that requires only one pass per row/column and each lifting step requires separate multiplication units [20,21]. Provide unique scalable architectures using the line-based lifting scheme method similar to that employed in this thesis to reduce the memory requirements for performing the 2-D DWT [22,23].

The role of the compression is to reduce bandwidth requirements for transmission \& memory requirements for storage of all forms of data as it would not be practical to put images, audio, video alone on websites without compression [24,25]. Hardware implementation of any algorithm leads to speed up the processing time [26,27]. Compacting VLSI and FPGA to realize the implemented hardware is an efficient architecture $[28,29,30]$.

Hardware filter processor was implemented via the convolution operation with the support of software $[31,32]$. The hardware architecture of image processing system based on time sharing to synchronize the processing time $[33,34]$. The convolution processor is a linear operation using low pass and high pass filters to implement 2D-DWT [35,36]. Programmable Logic Devices (PLD and EPLD) are used for real time image processing to minimize the processing time as possible, also Programmable Logic Devices Digital Signal Processor Architecture (PLD+DSP) are applied [37,38].

It's a big task when searching or research and investigation on the subject has been trying to beneficiation by many researchers where they are provided methods and techniques cannot be underestimated in the subject of image compression. It has been the transition from traditional to modern technologies and high efficiency of applied technologies. Here it must be pointed to a very important point is how to manage tasks efficiently applied in the program leads to an influential actor and have effective results of the work.

\section{Literature Review}

Many works and paper are published related to this field, many of these works are explained previously so this section will concentrated on the direct hardware of image compression.

Chao Cheng, and Keshab K. Parhi (2008) presented a high speed, very large-scale systematic application of the discrete wavelet transform based on parallel finite impulse response filter with the hardware 
structures implementation. The system output can be improved by a factor of four per proposed approach. On the other hand the cost of the equipment increases by a factor of three. Parallel and efficient finite impulse response filter structure is improved to accelerate the processing speed of discrete wavelet transform and to control the cost of the required equipment's simultaneously [31].

R.Lavanya and Saranya B. (2010) designed and implemented folded wavelet filters with the characteristics of high speed and low complexity architecture. The implemented approach aimed to improve the reconfigurable architecture speed. This is a method of analysis to improve communication between tasks and in addition between dependencies between tasks that will reduce processing time and the overall of the required communications [32].

Takkiti Rajashekar Reddy, Rangu Srikanth (2011), developed basic discrete wavelet transform image processing system using Spartan3 Xilinx Field Programmable Gate Array device via Xilinx's integrated development tools. Two different discrete wavelet transform hardware architectures have been implemented in this embedded system. One of them is the direct execution of the two dimensional discrete wavelet transform cascade of two processes of on dimensional discrete wavelet transform. Another approach is implementing two dimensional discrete wavelet transform with optimized control and architecture [33].

Husain.K.Bhaldar et al. (2012) represented high speed discrete wavelet transform for hardware implementation using 5/3 wavelet data for image compression applications. Voice compression is another application of Wavelets which reduces the transmission time in mobile applications. This work as a main objective was demonstrated that a big reduction of complexity with excellent efficiency can be obtained by implementing the multiplying via discrete wavelet transform implementation in FPGA using this wavelet filters. DWT generates an analysis of multispectral that allows for an invariable interpretation of the image scale [34].

Khamees Khalaf Hasan et al. (2014) proposed a multilevel transform of discrete wavelet decomposition which is a flexible hardware architecture and directly apply for image compression. VHDL methodolody is applied to analysis and synthesis discrete wavelet transform decomposition architecture which can be implemented via Field Programmable Gate Array device. This makes each image size can be decomposition for the required level. A simple Harr wavelet mask are used in this approach to avoid complexity of the computation. The approach can be applied for wired and wireless applications [35].

Mr. Hemantkumar H. Nikhare, Prof. Ashish Singhadia (2015), explained the very large scale integration architectures for the application of two dimensional discrete wavelet transform. VHDL is applied to implement the required multiplier. Two type of multiplier are designed and implemented to perform this approach. Then a comparison process is applied to measure the energy consumption. The experimental results illustrated that Radix 4 multiplier have power reduction of $22.9 \%$ but the conventional radix 2 multiplier and almost $50 \%$ reduction of power [36].

Ms. Dhrisya, Mr. V Lakshmipathi (2016) explained that discrete wavelet transform plays an important part in many fields such as; signal compression, signal analysis and computer vision. Discrete wavelet transform required more memory for storing the intermediate computational results especially when it applied to image processing. This work analyzed the complexities and computational time of the Lifting based two discrete wavelet transform. This approach tend to developing a new algorithm of implementing pipeline architecture that is able of getting multiple data streams suitable for application in image and video processing which is require a real time processing [37].

\section{Hardware System Architecture}

\section{Methodology}

The hardware implemented system concentrated on introducing the Raspberry Pi which has the ability to operate in real time. Many generations of Raspberry Pi have been manufactured, started from first generation (1G) applied at 2012 to the last or third generation (3G) applied at 2016 [38].

The Raspberry Pi is standalone microcomputer system which was designed at United Kingdom, University of Cambridge University [38]. The main purpose of these devices is for teaching of computer science and information technology at schools [39]. The Raspberry pi is integrated from $700 \mathrm{MHz}$ ARM11 co-processor [40], $512 \mathrm{MB}$ RAM on model B+ whereas $256 \mathrm{MB}$ RAM on model A and Broadcom video core IV [34]. Raspberry Pi 3 is a microcomputer and it have the ability for real time working which can be control all jobs and operations of the implemented. The architectural design of Raspberry Pi is shown in Fig.1 [41].

Fig. 2 shows the implemented hardware system using Raspberry $\mathrm{Pi}$, which have the ability to monitor and control all the jobs and functions at real time. In this case all image operations (including image compression) can be implemented and run at real time without any indicated delay. 


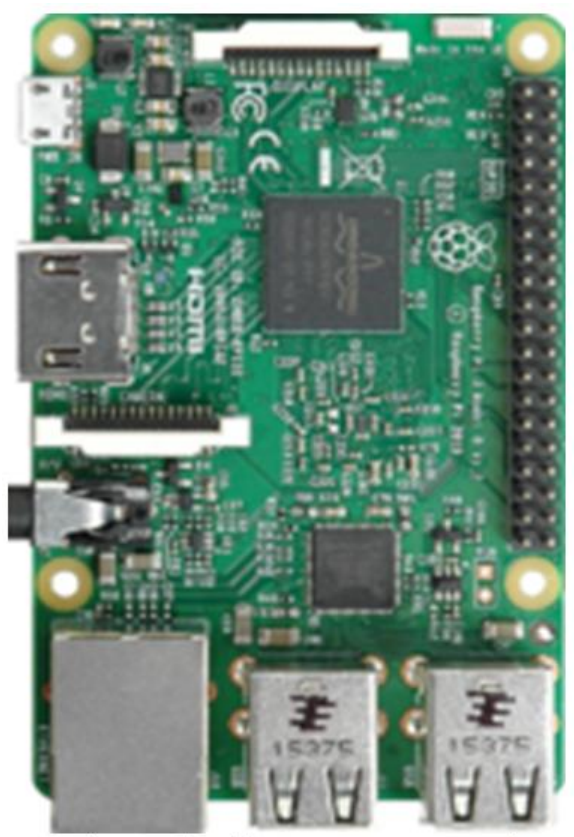

Figure 1 Raspberry Pi architecture

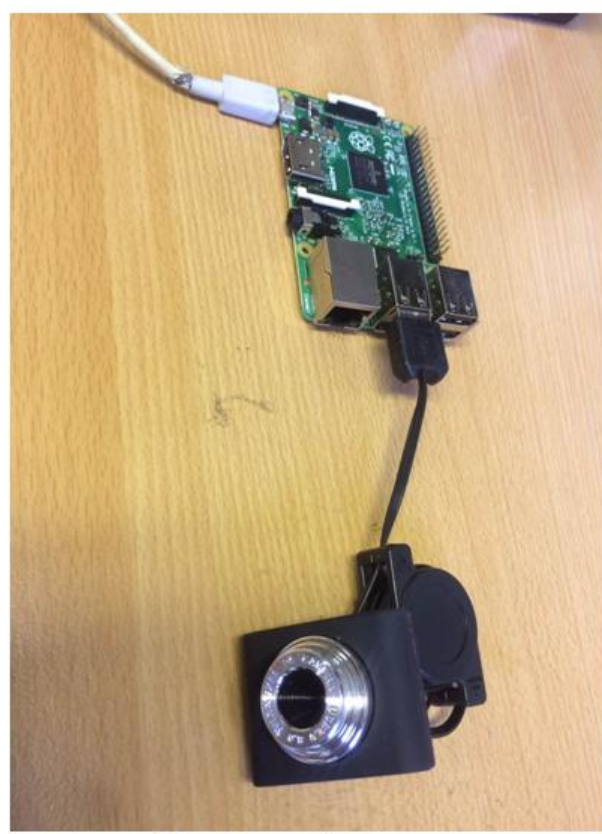

Figure 2 Raspberry Pi implemented system

\section{Fast 2D-DWT Structure}

Discrete Wavelet Transform (DWT) is a powerful signal processing tool that has recently gained widespread acceptance in the field of digital image processing. The multiresolution analysis provided by the DWT addresses the shortcomings of the Fourier Transform and its derivatives. The direct application of 2DDWT is image compression, so this structure implement the image compression via 2D-DWT.

The simple implementation of 2D-DWT is via applying low pass filter (LPF) and high pass filter (HPF) on rows and columns respectively. In this case we get four bands: low-low band (LL-band) via applying LPF twice on rows and columns, low-high band (LH-band) via applying LPF on rows and HPF on columns, high-low band (HL-band) via applying HPF on rows and LPF on columns and high-high band (HH-band) via applying HPF twice on rows and columns. Fig. 3 indicates the three levels of 2D-DWT applied on an image, in which the final image will be $(1 / 4 * 1 / 4 * 1 / 4)$ i.e. $(1 / 64)$ of the original image. On other word if we have the square image of size $(1024 * 1024)$ that leads to a good image resolution, in this case of applying third level 2D-DWT we reach the size of $(8 * 8)$ i.e. the third level of $2 \mathrm{D}-\mathrm{DWT}$ will be of size $(128 * 128)$.

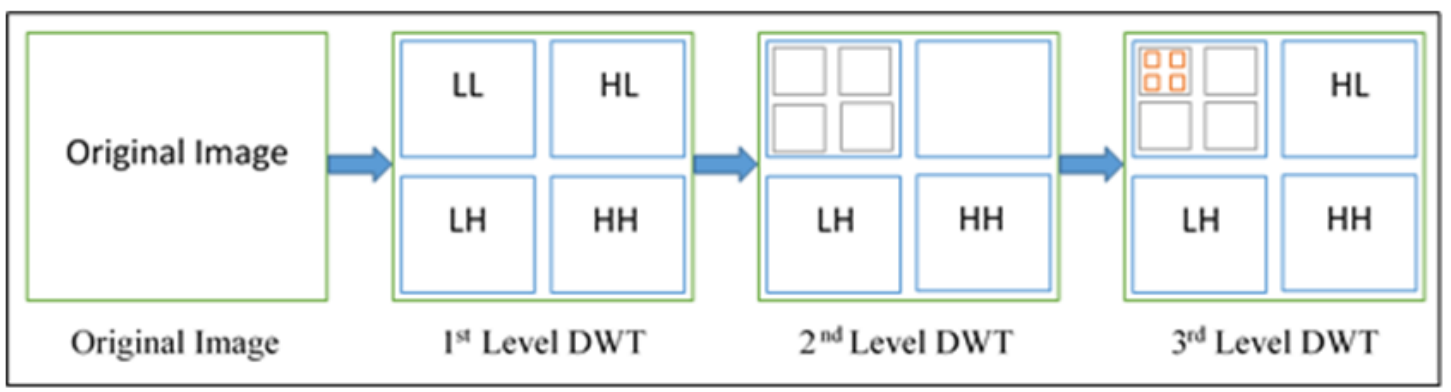

Figure 3 Three levels of 2D-DWT of an image

\section{Implemented 2D-DWT}

At the beginning of 2D-DWT architecture, try to explain the mathematical model of 2D-DWT. Suppose the size of the input square image is $\mathrm{N}^{*} \mathrm{~N}$, so the size after applying first level $2 \mathrm{D}$-DWT is $\mathrm{N} / 2 * \mathrm{~N} / 2$, the size after applying second level 2D-DWT is N/4*N/4 and the size after applying third level 2D-DWT is N/8*N/8.

Let $\mathrm{n} 1$ represents the row index and $\mathrm{n} 2$ represents the column index of the input image.

Let $\mathrm{k} 1$ represents the row index and $\mathrm{k} 2$ represents the column index of the output image.

Let $x(n 1, n 2)$ represents the input image, and represents $X(k 1, k 2)$ the output image.

Let the LPF impulse response is defined as hLPF.

Let the HPF impulse response is defined as hHPF. 
The LL-band of 2D-DWT is given below:

$X_{L L}(k 1, k 2)=\sum_{n 1=0}^{N-1} \quad \sum_{n 2=0}^{N-1} x(2 k 1-n 1,2 k 2-n 2) h_{L P F 1}(\mathrm{n} 1) h_{L P F 2}(\mathrm{n} 2)$

The LH-band of 2D-DWT is given below:

$X_{L H}(k 1, k 2)=\sum_{n 1=0}^{N-1} \quad \sum_{n 2=0}^{N-1} x(2 k 1-n 1,2 k 2-n 2) h_{L P F 1}(\mathrm{n} 1) h_{H P F 2}(\mathrm{n} 2)$

The HL-band of 2D-DWT is given below:

$X_{H L}(k 1, k 2)=\sum_{n 1=0}^{N-1} \quad \sum_{n 2=0}^{N-1} x(2 k 1-n 1,2 k 2-n 2) h_{H P F 1}(\mathrm{n} 1) h_{L P F 2}(\mathrm{n} 2)$

The HH-band of 2D-DWT is given below:

$X_{H H}(k 1, k 2)=\sum_{n 1=0}^{N-1} \quad \sum_{n 2=0}^{N-1} x(2 k 1-n 1,2 k 2-n 2) h_{H P F 1}(\mathrm{n} 1) h_{H P F 2}(\mathrm{n} 2)$

To get second level 2D-DWT X_LL $(\mathrm{k} 1, \mathrm{k} 2)$ will be the input of equation (1) and so on for the third level 2DDWT.

Each level of 2D-DWT required two sages: first stage concern to the system and flow control in which consists of two memory FIFOs separated by flip flop delay and the second stage consists of the structure implementation of 2D-DWT. To implement the above equations in a real three levels of 2D-DWT architecture, it is convenient to follow the following steps (fig.4):

Step1: the flow of pixels is controlled and it is divided into two parts; even pixels and odd pixels.

Step2: the even pixels is generated via memory FIFO1.

Step3: the odd pixels is generated via the combination of memory FIFO2 with flip-flop FF1.

Step4: the even pixels flow via first low pass filter LPF1 to generate the low band.

Step5: the output of LPF1 flows via first low pass filter LPF2 to generate the low-low band (LL-band).

Step6: the output of LPF1 flows via first high pass filter HPF1 to generate the low-low band (HL-band).

Step7: the odd pixels flow via first low pass filter HPF2 to generate the high band.

Step8: the output of HPF2 flows via third low pass filter LPF3 to generate the high-low band (HL-band).

Step9: the output of HPF2 flows via third high pass filter HPF3 to generate the high-high band (HH-band).

Step10: the output of LPF2 (LL-band) will pass to the second level 2D-DWT.

Step11: the output of LPF5 (LL-LL-band) will pass to the third level 2D-DWT.

Step12: the output of LPF8 (LL-LL-LL-band) will generate the third level 2D-DWT.

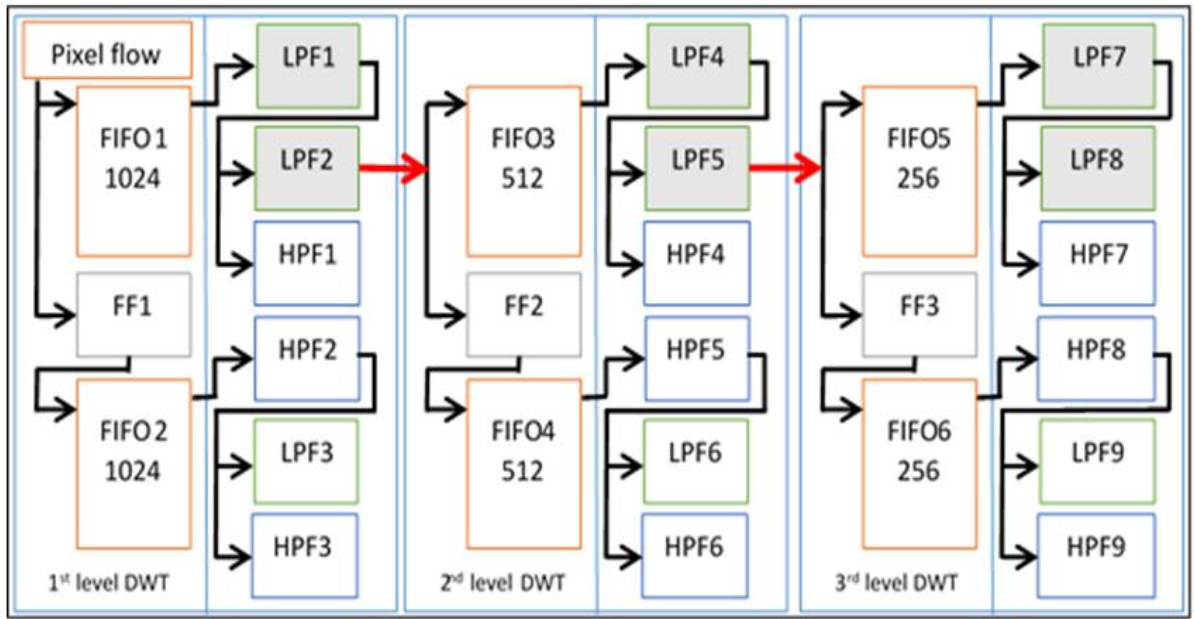

Figure 4 three levels 2D-DWT architecture

\section{Results and discussions}

As it is shown in figure 4 in each level of 2D-DWT we have two memory FIFO, one flip-flop, three low pass filter and three high pass filter. But for real working when we pass from one level to the other we need only two low pass filter in each level, so the overall active filter to generate the third level 2D-DWT is six low pass filters as shown in table 1. It is clear from this table that the required number of operations (number of cycles) is 1032192 . 
Table 1 the used components of 2D-DWT

\begin{tabular}{|l|l|l|l|l|l|l|l|l|l|l|l|}
\hline & FIFO & FF & LPF & HPF & $\begin{array}{l}\text { Active } \\
\text { filters } 1^{\text {st }} \\
\text { level }\end{array}$ & $\begin{array}{l}\text { Active } \\
\text { filter } \\
2^{\text {nd }} \text { level }\end{array}$ & $\begin{array}{l}\text { Active } \\
\text { filter } \\
3^{\text {rd }} \text { level }\end{array}$ & $\begin{array}{l}\text { Overall } \\
\text { Active } \\
\text { filters }\end{array}$ & MSE & $\begin{array}{l}\text { PSNR } \\
\text { Opumber of } \\
\text { Operations }\end{array}$ \\
\hline $1024 * 1024$ & 2 & 1 & - & - & & & & & & & \\
\hline $1024 * 512$ & - & - & 1 & 1 & 1 & & & 1 & & & 524288 \\
\hline $512 * 512$ & 2 & 1 & 2 & 2 & 1 & & & 1 & 40.86 & 30.02 & 262144 \\
\hline $512 * 256$ & - & - & 1 & 1 & & 1 & & 1 & & & 131072 \\
\hline $256 * 256$ & 2 & 1 & 2 & 2 & & 1 & & 1 & 56.40 & 30.62 & 065536 \\
\hline $256 * 128$ & - & - & 1 & 1 & & & 1 & 1 & & & 032768 \\
\hline $128 * 128$ & - & - & 2 & 2 & & & 1 & 1 & 94.14 & 28.39 & 016384 \\
\hline Total & 6 & 2 & 9 & 9 & 2 & 2 & 2 & 6 & & & 1032192 \\
\hline
\end{tabular}

Fig. 5 shows that the preceding process required half number of operation of the previous process, and at the end we get $1 / 64$ of the original image means the compression ratio is $1 / 64$. On the other hand you can see that the total number of operation of the third level 2D-DWT is 1,032,192. According to the Raspberry pi is integrated from $700 \mathrm{MHz}$, this leads to $1 / 700=1.42857 \mathrm{~ns}$ for each cycle. In this case the implementation of third level 2D-DWT required $0.00147456 \mathrm{~s}$ or $1474.56 \mu \mathrm{s}$ which is so enough for real time image processing. The error measures indicated that with the increase of the compression ratio or increase the level of 2D-DWT, then the values of MSE increased, and the values of PSNR decreased as shown in fig.6.
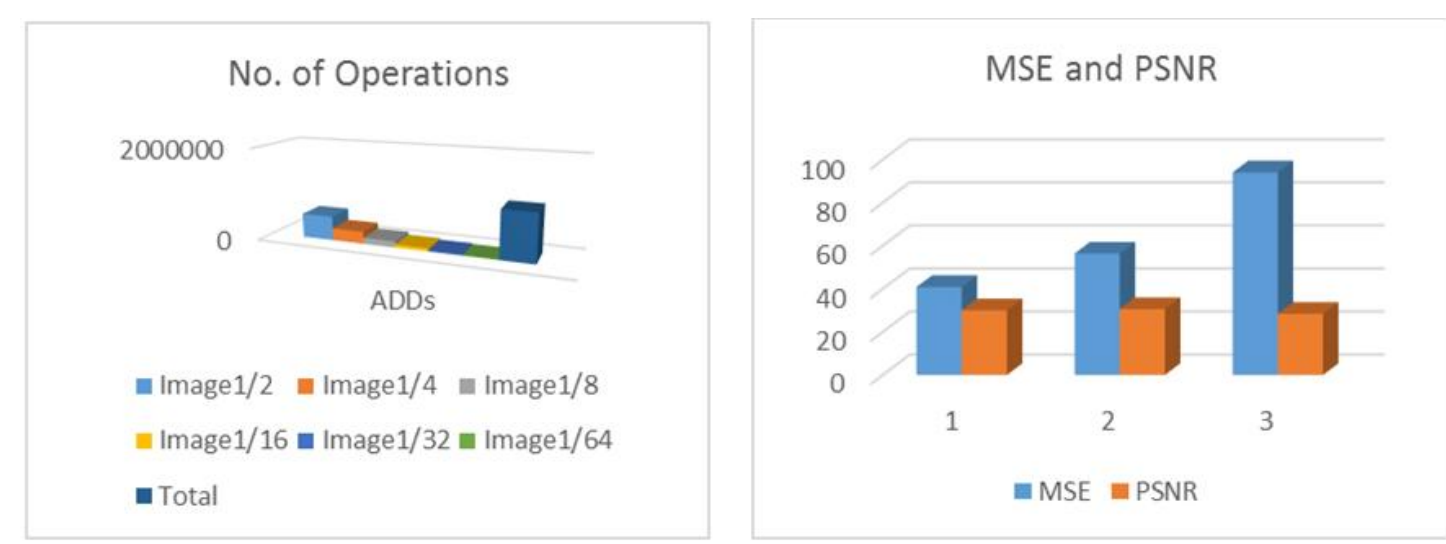

Figure 5 no. of operations in each step of 2D-DWT Figure 6 MSE and PSNR

The application of natural view image of $1024 * 1024$ size, in which you can see the application of 1 st level, 2 nd level and 3rd level 2D-DWT of the original image as shown in figures 7, 8 and 9 respectively. At these figures also you can see the values of MSE and PSNR.

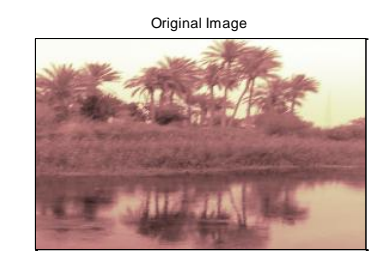

Original Image
Normalized Histogram
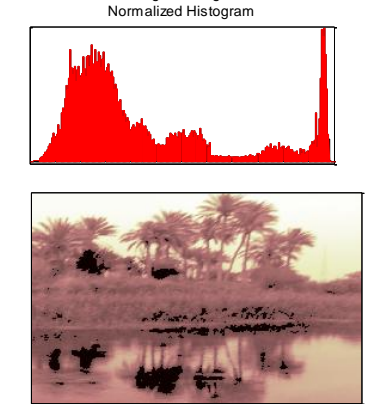

Compressed Image

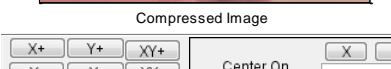

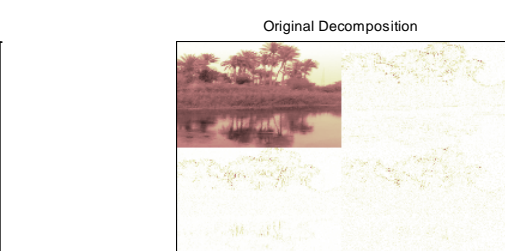
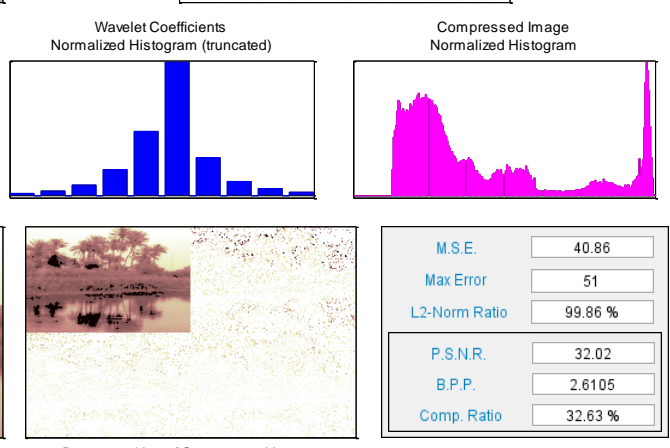

Decomposition of Compressed Image

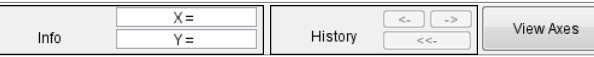

Figure 7 applied of $1^{\text {st }}$ level 2D-DWT

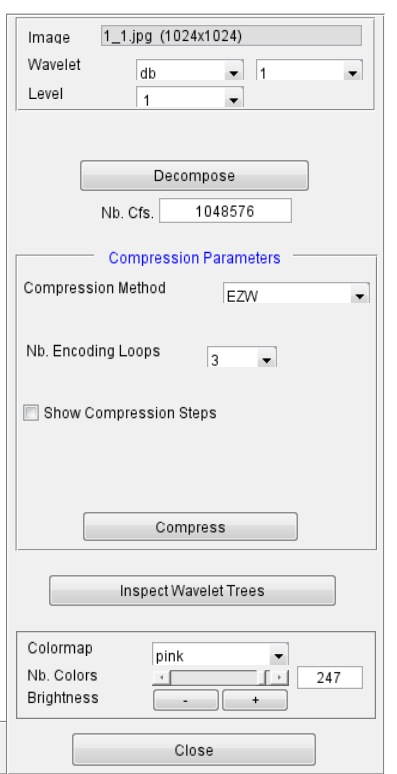

(a) 

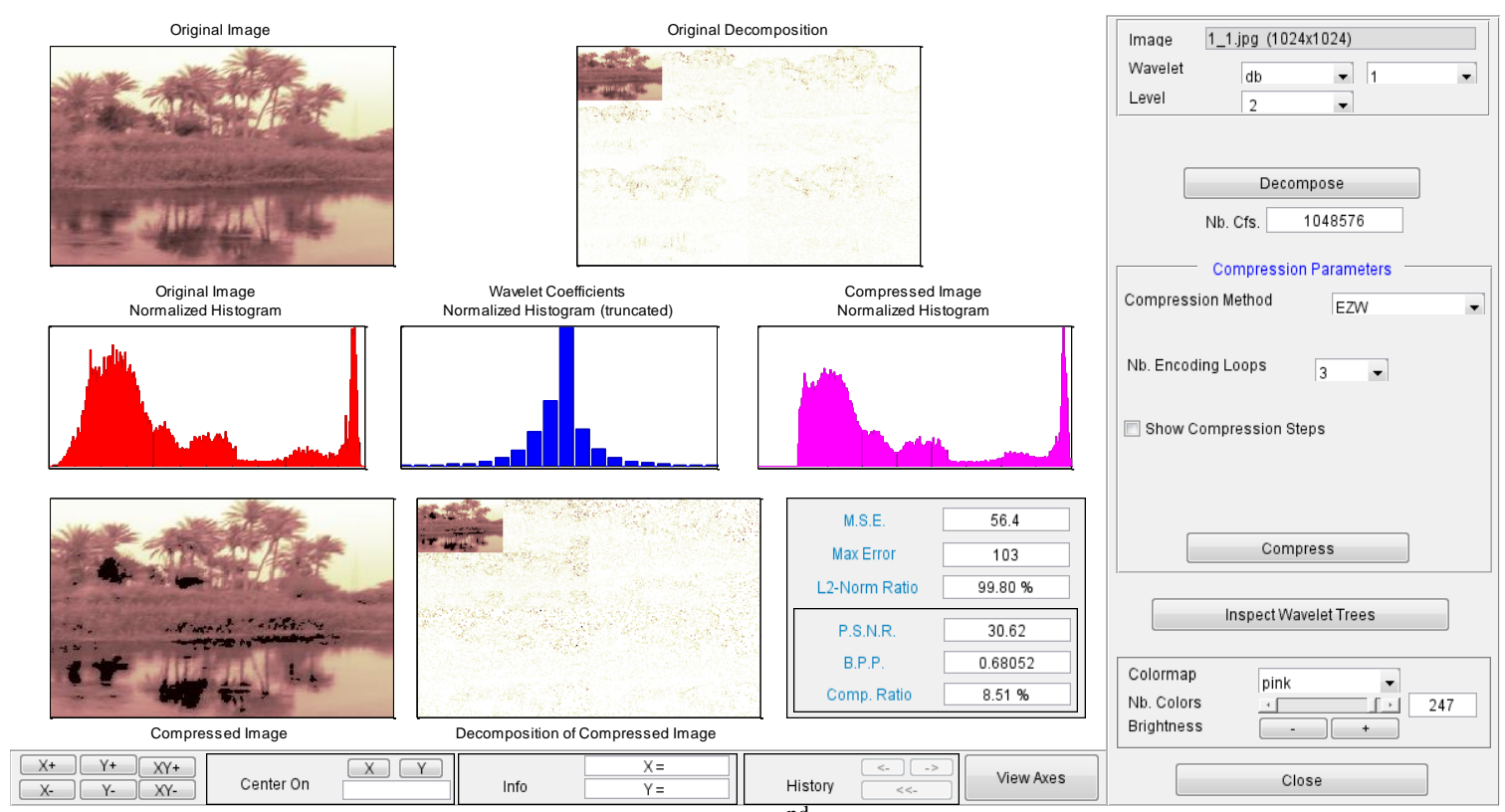

Figure 8 applied of $2^{\text {nd }}$ level 2D-DWT

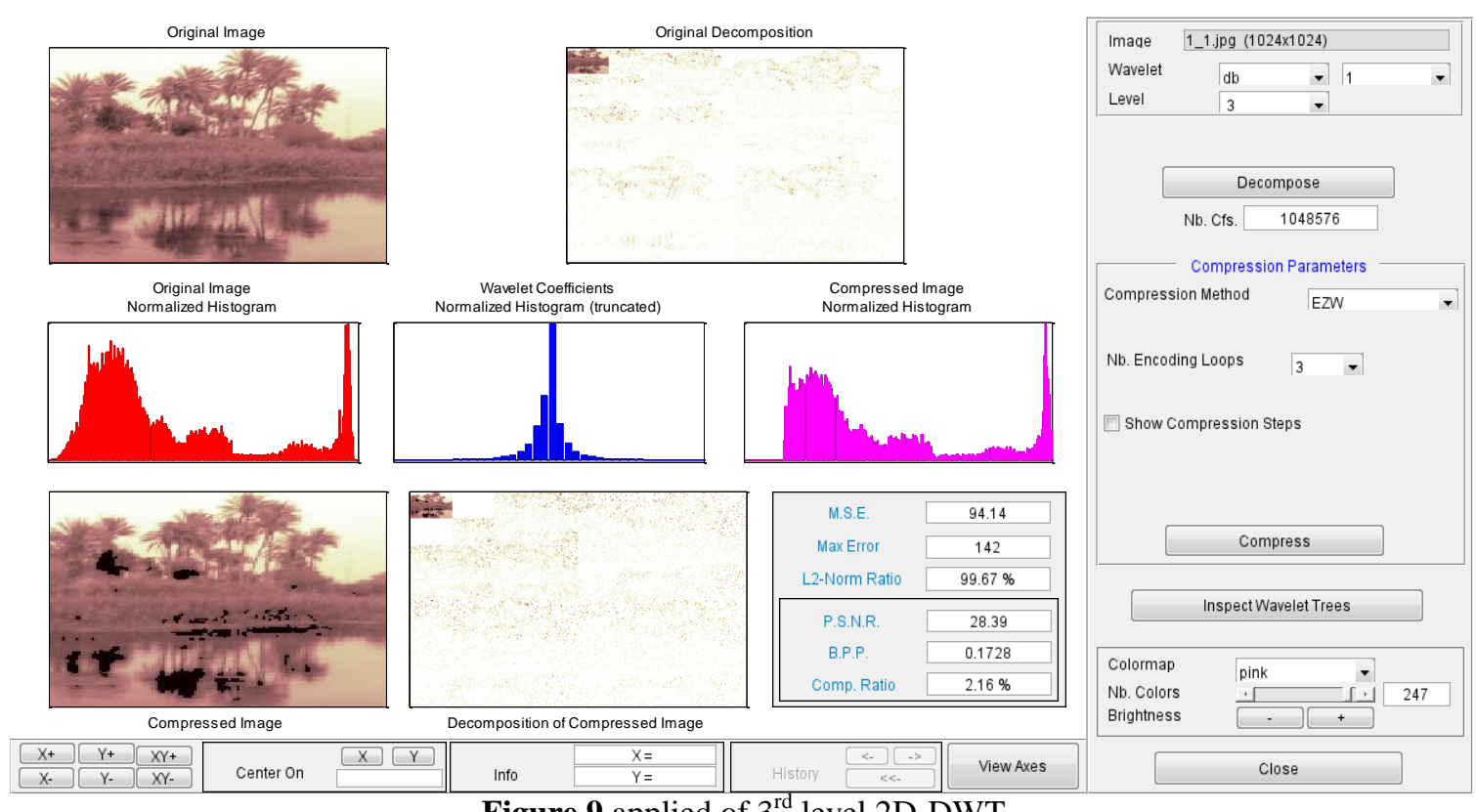

Figure 9 applied of $3^{\text {rd }}$ level 2D-DWT

\section{Conclusion}

Image and video compression have wide range of applications and there are many techniques that applied to perform the compression, one of the effective technique is discrete wavelet transform. In this work, image compression via two dimensional discrete wavelet transform, is implemented using Raspberry Pi hardware device (microcomputer), this will accelerate the processing speed. Raspberry $\mathrm{Pi}$ is a microcomputer manufactured in UK in 2012 for teaching of computer and information technology subjects. The implemented algorithm is concentrated on third level 2D-DWT in which required six active sequential filters (LPF and HPF), two filters in each level. The required number of operations is 1032192, in which the required processing time is $1474.56 \mu$ s i.e. it is able to be real time processing.

\section{References}

[1]. M. Rabbani, and P.W. Hones, Digital Image Compression Techniques, SPIE Opt. Eng. Press, Bellingham, Washington, 1991.

[2]. M. Antonini, M. Barlaud, P. Mathieu, and I. Daubechies, "Image coding using wavelet transform, "IEEE Trans. Image Processing, vol. 1, pp. 205-220, April 1992.

[3]. Win-Bin Huang, Yuan-Jui Chang, Alvin W.Y. Su and Yau-Hwang Kuo, "VLSI Design of A DWT/Modified Efficient SPIHT Based Image Codec", ICICS,-PCM 11-18 December Singapore 2003. 
[4]. M. W. Marcellin, M. J. Gormish, A. Bilgin and M. Boliek, “An overview of JPEG-2000”, Proc. of Data Compression Conference, Snowbird, Utah, pp. 523-541, Mar. 2000.

[5]. ISO/IEC 15444-1:2000(E), Information technology - JPEG-2000 Image Coding System - Part 1: Core Coding System.

[6]. P. Meerwald, R. Norcen, and A. Uhl, "Parallel JPEG2000 image coding on multiprocessors," in Parallel and Distributed Processing Symposium., Proceedings International, IPDPS 2002, Abstracts and CD-ROM, Ft. Lauderdale, FL, 2002, pp. 2-7.

[7]. S. Mallat, "A theory for multi resolution signal decomposition: the wavelet representation," IEEE Transactions on Pattern Anal. Machine Intell., Vol.11, pp.674-693, July 1989.

[8]. M. Vishwanath, "The Recursive Pyramid Algorithm for the Discrete Wavelet Transform," IEEE Trans. on Signal Processing, vol. 42, no. 3, pp.673-676, Mar 1994.

[9]. M. Jeyaprakash, "FPGA Implementation of Discrete Wavelet Transform (DWT) for JPEG 2000", International Journal of Recent Trends in Engineering, Vol. 2, No. 6, November 2009.

[10]. S. Mallat, A Wavelet Tour of Signal Processing, 3rd ed. Academic Press, 2008.

[11]. P. Meerwald, R. Norcen, and A. Uhl, "Parallel JPEG2000 image coding on multiprocessors," in Parallel and Distributed Processing Symposium., Proceedings International, IPDPS 2002, Abstracts and CD-ROM, Ft. Lauderdale, FL, 2002, pp. 2-7.

[12]. Xin Tian, Lin Wu , Yi-Hua Tan "Efficient Multi-Input/Multi-Output VLSI Architecture for Two-Dimensional Lifting-Based Discrete Wavelet Transform," IEEE Transactions on Computers, vol. 60, no. 8, August 2011.

[13]. K. Oweiss, "A Systems Approach for Data Compression and Latency Reduction in Cortically Controlled Brain Machine Interfaces,” IEEE Tran. on Biomed. Eng., vol. 53, no. 7, pp. 1364-1377, 2006.

[14]. Awais M. Kamboh, Andrew Mason and Karim Oweiss, "A High-Yield Area-Power Efficient DWT Hardware for Implantable Neural Interface Applications", Proceedings of the 3rd International

[15]. IEEE EMBS Conference on Neural Engineering Kohala Coast, Hawaii, USA, May 2-5, 2007

[16]. M. Martina, and G. Masera, "Low-complexity, efficient 9/7 wavelet filters VLSI implementation," IEEE Trans. on Circuits and Syst. II, Express Brief vol. 53, no. 11, pp. 1289-1293, Nov. 2006.

[17]. ie Guo, Ke-yanWang, Cheng-keWu and Yunsong Li, Efficient FPGA Implementation of Modified DWT for JPEG2000, 978-14244-2186-2/08 2008 IEEE.

[18]. G. Savaton, E. Casseau, and E. Martin. "High Level Design and Synthesis of a Discrete Wavelet Transform Virtual Component for Image Compression". In IP Based Design 2000, Dec. 2000.

[19]. P. Mohaniah, P. Sathyanarayana, A. S. Ram Kumar Reddy and A. Vijayalakshmi, "Design and Implementation of 3-D DWT for Video Processing Applications", International Journal of Advanced Electrical and Electronics Engineering, (IJAEEE), Volume-1, Issue-3, 2012.

[20]. A. Pande and J. Zambreno "A reconfigurable Architecture for secure multimedia delivery" in IEEE Transactions in 23rd international conference on VLSI design 2010.

[21]. Shah V.P., Younan N.H., Durbha S.S., et al.,"Feature Identification via a Combined ICA-Wavelet Method for Image Information Mining", IEEE Geoscience and Remote Sensing Letters, 7(1), pp. 18-22, 2010.

[22]. Kavish Seth and S. Srinivasan. "VLSI implementation of 2-D DWT/IDWT cores using 9/7-tap filter banks based on the nonexpansive Symmetric extension scheme". Proceedings of the 15th International Conference on VLSI Design, pp. 435-440, Jan, 2002 .

[23]. K. Kotteri, S. Barua, A. Bell, and J. Carletta, "A comparison of hardware implementations of the biorthogonal 9/7 DWT: convolution versus lifting," IEEE Transactions on Circuits and Systems II, vol. 52, no. 5, pp. 256-260, May 2005.

[24]. C. Huang, P. Tseng, and L. Chen. "Efficient VLSI Architectures of Lifting-Based Discrete Wavelet Transform by Systematic Design Method". National Taiwan University: Dept. of EE. IEEE, 2002.

[25]. C. T. Huang, P. C. Tseng, and L. G. Chen, "Generic RAM-based architectures for two-dimensional discrete wavelet transform with linebased method," IEEE Trans. on Circuits and Systems, vol. 1, pp. 363- 366, 2002.

[26]. Wang.C, Gan .W.S, (2007),"Efficient VLSI architecture for lifting based discrete wavelet packet transform,”, IEEE Trans. Circuits and Systems-II: express briefs, Vol.54.no. 5, pp.422-426.

[27]. KARTHIKEYAN A., SARANYA P., JAYASHREE N., “An Efficient VLSI Architecture for 3D DWT Using Lifting Scheme”, International Journal of Engineering Science and Innovative Technology (IJESIT) Volume 2, Issue 1, January 2013, pp.292-298.

[28]. A. Cohen, I. Daubechies, and J. Feauveau, "Bi-orthogonal bases of compactly supported wavelets", Comm. Pure Appl. Math., vol. 45, pp. 485-560, 1992.

[29]. M. Jeyaprakash; "FPGA Implementation of Discrete Wavelet Transform (DWT) for JPEG 2000", International Journal of Recent Trend in Engineering, Vol. 2, No. 6, November 2009.

[30]. M. Martina, and G. Masera, \|Multiplierless, folded 9/7-5/3 wavelet VLSIarchitecture, IIEEE Trans. on Circuits andsyst. II, Express Brief vol. 54, no. 9, pp. 770-774, Sep. 2007.

[31]. K. Andra, C. Chakrabarti, and T. Acharya, "A VLSI architecture for lifting-based forward and inverse wavelet transform," IEEE Trans. Signal Process., vol. 50, no. 4, pp. 966-977, Apr. 2002.

[32]. B. Rui, S. Lorenzo, L. Nozal and Muzhir Shaban Mohammed Al-Ani, "Digital Signal Processor Accelerator Board for Image Processing on VME Bus Based System", Machine Vision Application, Architectures, and System Integration 17-18 November 1992, Boston, Manssachusetts, USA, PP 85-90.

[33]. L. Nozal, S. Lorenzo, B. Rui and Muzhir Shaban Mohammed Al-Ani, "Hardware Structure + Digital Signal Processing on Real Time", International Conference on Industrial Electronics, Control, Instrumentation and Automation, 9-13 November 1992, San Diego, California, USA, PP 1397-1402.

[34]. Muzhir Shaban Mohammed Al-Ani and Santiago Lorenzo, "System Implementation of LUT FFT", 6th Mediterranean Electrotechnical Conference (MELECON'91), 22-24 May, 1991, Yugoslavia.

[35]. Muzhir Shaban Mohammed Al-Ani, S. Lorenzo, and L. Nozal, "Fast 2D Convolution Filter Based on LUT FFT", IEEE International Symposium on Industrial Electronics, 25-27 May, 1992, Chain, PP 446-449.

[36]. Muzhir Shaban Mohammed Al-Ani, S. Lorenzo L. Nozal and B. Rui, "FFT LUT for Image Processing", The International Society for Optical Engineering (SPIE'92), Section of Algorithms Technique and Active Vision, 15-20 November 1992, USA, PP 121-129.

[37]. L. Nozal, S. Lorenzo, B. Rui and Muzhir Shaban Mohammed Al-Ani, "Pipe-Line Programmable Logic Device (PLD) A New Solution for Image Processing", SICE'91 Conference, 17-19 July, 1991, Yonezawa, Japan, PP 1097-1100.

[38]. L. Nozal, S. Lorenzo, B. Rui and Muzhir Shaban Mohammed Al-Ani, "A New Vision System Programable Logic Devices Digital Signal Processor Architecture (PLD+DSP)", International Conference on Industrial Electronics Control and Instrumentation (IECON'91)", 28 October - 1 November, 1991, Japan, PP 2014-2018. 
[39]. L. Nozal, S. Lorenzo, B. Rui and Muzhir Shaban Mohammed Al-Ani, "Real Time and Low Cost Image Processing Architecture Based on Programmable Logic Device (PLD)", Intelligent for Mechanical System, Proceedings IROS '91, 3-5 November, 1991, Osaka, Japan, PP 279-284.

[40]. Chao Cheng, and Keshab K. Parhi, "High-Speed VLSI Implementation of 2-D Discrete Wavelet Transform Methodology", IEEE Trans. On Signal Processing, Vol. 56, No. 1, January 2008 pp.393-403

[41]. R.Lavanya and Saranya B., "High Speed, Low Complexity, Folded, Polymorphic Wavelet Architecture Using Reconfigurable Hardware", International Journal of Advanced Science and Technology Vol. 18, May, 2010, pp.23-29.

[42]. Takkiti Rajashekar Reddy, Rangu Srikanth, "Hardware Implementation of DWT for Image compression using SPIHT Algorithm", International Journal of Computer Trends and Technology, volume2, Issue2, 2011

[43]. Husain.K.Bhaldar, V.K.Bairagi and R.B.Kakkeri, "Hardware Design of 2-D High Speed DWT by using Multiplierless 5/3 Wavelet Filters", International Journal of Computer Applications (0975 - 8887) Volume 59- No.17, December 2012 pp.42-46

[44]. Khamees Khalaf Hasan, Umi Kalthum Ngah, Mohd Fadzli Mohd Salleh, "Hierarchical Hardware Architecture of Discrete Wavelet Transform For Image Compression", International Journal of Computer Science and Information Technology Research, Vol. 2, Issue 4, pp: (232-241), Month: October - December 2014.

[45]. Mr. Hemantkumar H. Nikhare, Prof. Ashish Singhadia, "A Detailed Review on Architectures for 2-DWT by using Radix-4 Booth Multiplier", International Journal of Innovative Research in Electrical, Electronics, Instrumentation and Control Engineering Vol. 3, Issue 3, March 2015.

[46]. Ms. Dhrisya, Mr. V Lakshmipathi, "Efficient Multiplier Design for DWT through Lifting Scheme", International Journal for Research in Applied Science \& Engineering Technology (IJRASET), Volume 4 Issue III, March 2016.

[47]. J. D. Brock, R. F. Bruce, and M. E. Cameron, "Changing the world with a Raspberry Pi," J. Comput. Sci. Coll., vol. 29, no. 2, pp. 151-153, Dec. 2013.

[48]. P. Abrahamsson, S. Helmer, N. Phaphoom, L. Nicolodi, N. Preda, L. Miori, M. Angriman, J. Rikkila, X. Wang, K. Hamily, and S. Bugoloni, "Affordable and Energy-Efficient Cloud Computing Clusters: The Bolzano Raspberry Pi Cloud Cluster Experiment," 2013 IEEE 5th Int. Conf. Cloud Comput. Technol. Sci., pp. 170-175, Dec. 2013.

[49]. R. Heeks and A. Robinson, "Emerging Markets Ultra-Low- Cost Computing and Developing Countries," Commun.ACM, vol. 56, no. 8, pp. 22-24, 2013.

[50]. E. Upton and G. Halfacree, Raspberry Pi user guide. John Wiley \& Sons, 2014. 